\title{
Mechanism of $r$-mode stability in young rapidly rotating pulsars
}

\author{
E.E. Kolomeitsev ${ }^{1, a}$ and D.N. Voskresensky ${ }^{2}$ \\ 1 Matej Bel University, SK-97401 Banska Bystrica, Slovakia \\ 2 National Research Nuclear University (MEPhI), 115409 Moscow, Russia
}

Received: 29 September 2014

Published online: 9 December 2014

(C) The Author(s) 2014. This article is published with open access at Springerlink.com Communicated by D. Blaschke

\begin{abstract}
We demonstrate that stability of $r$-modes in young rapidly rotating pulsars might be explained if one takes into account strong medium modifications of the nucleon-nucleon interaction because of the softening of pionic degrees of freedom in dense nucleon matter. Presence of the efficient direct Urca processes is not required. Within our model the most rapidly rotating observed young pulsar PSR J0537-6910 should have mass $\geq 1.8 M_{\odot}$.
\end{abstract}

Within the standard scenario of the neutron star (NS) formation in the supernova explosion [1] the NS is typically formed rapidly rotating with an initial rotation frequency $\nu_{\text {in }} \sim 1 \mathrm{kHz}$. However, the majority of young pulsars $\left(<10^{5} \mathrm{y}\right.$ of age $)$ have rotation frequencies less than $10 \mathrm{~Hz}$, and the fastest young pulsar observed so far is PSR J0537-6910 [2] with $\nu_{\max }^{\text {young }}=62 \mathrm{~Hz}$ and age $5 \mathrm{ky}$. An efficient mechanism allowing to decelerate the rotation of a neutron star already at an early stage of its evolution concerns the $r$-mode instability predicted in $[3,4]$, see also review [5]. The $r$-mode oscillations lead to the emission of gravitational waves, which carry away most part of the initial angular momentum of a star and the star rotation decelerates rapidly. The $r$-mode amplitude would grow exponentially for any rotation frequency, if not a damping, because of a viscosity of warm neutron star matter [6]. The typical temperature in the interior of a pulsar of age $\sim 5 \mathrm{ky}$ is $\sim 2 \cdot 10^{7}-5 \cdot 10^{8} \mathrm{~K}$, depending on the mass of the star and the cooling scenario, see [7]. However, the $r$-mode instability proves to be strongest at higher temperatures $T \sim 10^{9} \mathrm{~K}$. Hence, young pulsars have necessarily passed through an instability phase during their early history. Thus, to verify the $r$-modes instability scenario for the star-rotation braking one should demonstrate that after the instability phase the pulsar frequencies remain larger than $\nu_{\max }^{\text {young }}$. Different mechanisms for suppression of the instability were studied. Most attempts were spent to find appropriate arguments to increase the values of shear and bulk viscosities. Finally, it was concluded, e.g. see [5], that the minimum value of the frequency at the $r$-mode stability boundary $\nu_{c}(T)$ is smaller than the value $\nu_{\max }^{\text {young }}$, if one

\footnotetext{
a e-mail: E.Kolomeitsev@gsi.de
}

uses standard dissipation mechanisms only. The problem proved to be even more serious after ref. [8] demonstrated that the lepton shear viscosity should be strongly suppressed by in-medium polarization effects, compared to the result of the previous calculation [9].

In this letter we investigate the $r$-mode stability of young pulsars taking into account polarization effects in nuclear matter, among which the most efficient is the softening of pionic degrees of freedom with an increase of the nucleon density. This effect proves to be important in description of many phenomena in atomic nuclei, neutron stars, and heavy-ion collisions [10]. In particular, incorporating the above effects, the "nuclear medium cooling" scenario developed in $[7,11-17]$ allows to fit all existing data on the pulsar surface temperatures, including the recent data on the cooling of the young pulsar in Cassiopea A. We will incorporate mentioned effects in the $r$-mode dissipation mechanisms. Also we include new contributions, such as the neutrino shear viscosity in the neutrino trapping region and radiative bulk viscosity calculated with account for nucleon medium polarization effects.

The characteristic time scale of the $r$-mode amplitude evolution is given by

$$
\tau^{-1}=\tau_{\eta}^{-1}+\tau_{\zeta}^{-1}-\tau_{G}^{-1},
$$

where $\tau_{G}$ is the typical time of the gravitational radiation, $\tau_{\eta}$ denotes the relaxation time induced by the shear viscosity, $\tau_{\zeta}$ stands for the relaxation time induced by the bulk viscosity. The $r$-modes are unstable, if $\tau^{-1}<0$. The gravitational time for the most unstable mode, which oscillation frequency is related to the angular velocity of the 
pulsar $(\Omega=2 \pi \nu)$ as $\omega=4 \Omega / 3$, is equal to $[6]$

$$
\tau_{G}^{-1}=6.4 \cdot 10^{-2}[\mathrm{~Hz}] R_{6}^{7} \Omega_{4}^{6} \rho_{\mathrm{cen}} / \rho_{0},
$$

where $R_{6}=R /\left(10^{6} \mathrm{~cm}\right), \Omega_{4}=\Omega /\left(10^{4} \mathrm{~Hz}\right)$ and $\rho_{0}=$ $m_{N} n_{0}=2.63 \cdot 10^{14} \mathrm{~g} / \mathrm{cm}^{3}$ is the mass density of the nuclear matter at saturation, with $m_{N}=938 \mathrm{MeV}$ being the nucleon mass in vacuum. The central mass density of the star $\rho_{\text {cen }}$ depends on the neutron star mass. The damping times of the $r$-modes can be written as [6]

$$
\tau_{\eta}^{-1}=6.0 \cdot 10^{-5}[\mathrm{~Hz}]\left\langle\eta_{20}\right\rangle_{4} R_{6}^{-2} \rho_{0} / \rho_{\text {cen }},
$$

and

$$
\begin{aligned}
\tau_{\zeta}^{-1}= & 2.2 \cdot 10^{-7}[\mathrm{~Hz}] R_{6}^{4} \Omega_{4}^{4}\left\langle\zeta_{20}\left[1+0.86(r / R)^{2}\right]\right\rangle_{8} \\
& \times\left(\frac{M_{\odot}}{M}\right)^{2} \frac{\rho_{0}}{\rho_{\text {cen }}}
\end{aligned}
$$

where $\eta_{20}, \zeta_{20}$ stand for the shear and bulk viscosities measured in units of $10^{20} \mathrm{~g} /(\mathrm{cm} \cdot \mathrm{s})$. The angular brackets mean the averaging

$$
\langle\ldots\rangle_{n}=R^{-(n+1)} \int_{0}^{R}(\ldots) r^{n} \mathrm{~d} r .
$$

For the nucleon densities $n>0.6 n_{0}$ we exploit the HDD equation of state (EoS) constructed in [7] to be close to the realistic Akmal-Pandharipande-Ravenhall (APR) A18 $+\delta v+$ UIX $^{*}$ EoS [18] for densities $n \lesssim 4 n_{0}$. Contrary to the APR EoS, the HDD EoS is causal for all densities, producing the maximum mass $M_{\max } \simeq 2.05 M_{\odot}$, being in agreement with observations $[19,20]$. As for the APR EoS, the one-nucleon direct Urca (DU) processes with electrons, $n \rightarrow p+e+\bar{\nu}$, start to contribute only for densities $n>5 n_{0}$, i.e., for stars with masses $M>M_{c}^{\mathrm{DU}} \simeq 1.9 M_{\odot}$. Dependence of the effective nucleon mass on the nucleon density is parameterized [10] as $m_{N}^{*} \approx m_{n}^{*} \approx m_{p}^{*} \approx$ $\left[1-0.15\left(n / n_{0}\right)^{1 / 2}\right] m_{N}$. For the density $n \simeq 0.6 n_{0}$ we match the HDD EoS with the Friedman-PandharipandeSkyrme EoS [21] that we use for lower densities. As in all previous papers discussing $r$-mode instability, we will use a simplifying assumption of a homogeneous distribution of the temperature in the star core.

For calculations of different partial contributions to the neutrino emissivities and viscosities one often uses the free one-pion exchange (FOPE) model or, sometimes, the free nucleon-nucleon $(N N)$ cross-section corrected by Pauli blocking $[8,22]$. Alternatively we exploit here the $N N$ interaction constructed within the Fermi-liquid approach [23], wherein nucleon particle-hole excitations are taken into account explicitly while other processes are incorporated as a phenomenologically parameterized residual interaction. Pionic modes are soft $\left(m_{\pi} \ll m_{N}, m_{\pi}\right.$ is the pion mass). Therefore, they are treated explicitly on equal footing with the nucleon particle-hole modes. As the result, the main contribution to the $N N$ interaction at densities $n \gtrsim n_{0}$ is given by the medium one-pion exchange (MOPE), whereas the relative contribution of the residual interaction diminishes with increasing density because of polarization effects, see [10] and references therein. (a)

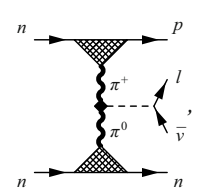

(b)

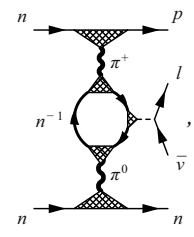

(c)

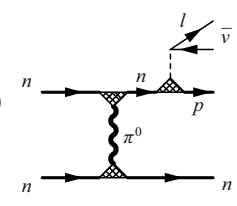

Fig. 1. Set of diagrams determining the MMU reactions.

Allowing for nucleon superfluidity in the star interior, for the neutron and proton ${ }^{1} \mathrm{~S}_{0}$ pairing gaps we use same parameterizations as in $[7,15-17]$ (set I shown in fig. 2 in [7]). The critical temperature of the neutron triplet pairing was argued [24] to be strongly reduced to the values $\sim 10^{8} \mathrm{~K}$ because of a medium-induced spin-orbit interaction. This choice has been exploited in the nuclear medium cooling scenario $[7,15-17]$ and we continue to use it here.

For calculations of the $r$-mode damping times $\tau_{\eta}$ and $\tau_{\zeta}$ we need shear and bulk viscosities. The bulk viscosity is presented as the sum of three contributions, the collisional term, the "soft-mode" term and the radiative term. The collisional term, determined mainly by $n n$ collisions is found to be small. The soft-mode contribution to the bulk viscosity is induced in pulsating medium by reactions generated by charged weak currents. The radiative bulk viscosity contributions prove to be of the same order as the soft-mode ones.

Within the "minimal cooling" scenario [25] in the absence of the DU reactions, for $M<M_{c}^{\mathrm{DU}}$, the most efficient cooling processes in a non-superfluid part of a NS are the modified Urca (MU) processes $N+n \rightarrow N+p+l+\bar{\nu}_{l}$ and $N+p+l \rightarrow N+n+\nu_{l}$, for $N=n, p$ and $l=e, \mu^{-}$. The soft-mode bulk viscosity due to the MU processes was studied in $[26,27]$, where the FOPE model of [22] was used for the description of the $N N$ interaction. The efficiency of so-calculated MU processes $\left(\sim 10^{6}\right.$ times less than that would be for the DU processes) is insufficient to stabilize the $r$-modes. Thus, the problem of the $r$-mode instability becomes severe for the stars with masses $M<M_{c}^{\mathrm{DU}}$.

In the case when the $N N$ interaction amplitude is mainly controlled by the soft-pion exchange, the MU matrix element should be replaced by the matrix element for the medium modified Urca (MMU) processes, which for densities $n \gtrsim n_{0}$ are mainly determined by the diagrams shown in fig. 1. Here the bold wavy line depicts the in-medium pion. The hatched vertices are dressed by $N N$ correlations. Calculations [11-14] show that the dominant contributions to the MMU rate come from the first two diagrams in fig. 1, whereas the third diagram, which would be a naive generalization of the corresponding $\mathrm{MU}$ (FOPE) contribution, gives only a small correction for $n \gtrsim n_{0}$. We use the same parameterization of the $N N$ interaction, degree of the pion softening, and contributions to the matrix element of the neutrino reactions as in $[7$, 15-17]. With these estimates, the bulk viscosity owing to the MMU reactions increases by three to four orders of magnitude for densities $n \sim 3 n_{0}$, in accordance with the corresponding increase of the neutrino emissivity of the MMU processes studied in [7,15-17]. For densities $n>n_{c}^{\pi}$, there may appear a pion condensate, as the consequence of enhancement of the pion softening with a density increase. 


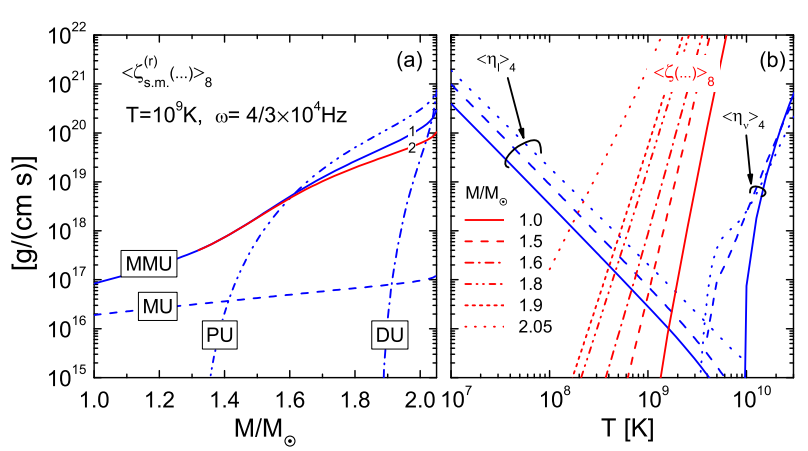

Fig. 2. Panel (a): Partial contributions to the bulk viscosity averaged over the star density profile, $\left\langle\zeta_{\mathrm{s} . \mathrm{m} .}^{\mathrm{r}}\left(1+0.86 r^{2} / R^{2}\right)\right\rangle_{8}$, from DU, MU, MMU, and PU reactions as functions of the NS mass. Line 1 shows the contribution of the MMU reaction in absence of the pion condensate and line 2, in its presence. $\mathrm{Nu}$ cleon pairing is not included. Panel (b): The profile-averaged lepton $\left\langle\eta_{l}\right\rangle_{4}$ and neutrino $\left\langle\eta_{\nu}\right\rangle_{4}$ shear viscosity terms, and the averaged total bulk viscosity $\left\langle\zeta\left(1+0.86 r^{2} / R^{2}\right)\right\rangle_{8}$, being calculated for the neutrino MMU reactions, plotted as functions of the temperature for several NS masses. Nucleon pairing is included. Calculations are done for $\omega=(4 / 3) \cdot 10^{4} \mathrm{~Hz}$.

We consider two possibilities: pion condensate appears at $n_{c}^{\pi}$, and it does not appear provided the pion softening saturates at higher densities. The contribution to the bulk viscosity owing to reactions on a charged pion condensate - pion Urca (PU) reaction, $n+\pi_{\mathrm{c}}^{-} \rightarrow n+l+\bar{\nu}_{l}$ - is included in the case when the condensate appears, at the critical density taken to be $n_{c}^{\pi}=3 n_{0}$, as in [7,15-17].

To illustrate the role of the pion softening, partial contributions to the bulk viscosity from different reactions averaged over the star density profile, $\left\langle\zeta_{\mathrm{s} . \mathrm{m} .}^{(\mathrm{r})}\left[1+0.86 r^{2} / R^{2}\right]\right\rangle_{8}$, are presented in fig. 2(a) for MU, MMU, PU, and DU reactions as functions of the NS mass for the temperature $T=10^{9} \mathrm{~K}$, but without account for pairing, and for the $r$-mode frequency $\omega_{4}=4 / 3$. We see that for $1 \lesssim M<$ $1.6 M_{\odot}$ the main contribution to $\left\langle\zeta_{\mathrm{s} . \mathrm{m}}\right\rangle_{8}$ comes from the MMU processes. For $M>1.6 M_{\odot}$ the PU process yields the dominant contribution. Note that $\left\langle\zeta_{\mathrm{s} . \mathrm{m} .}^{(\mathrm{DU})}\right\rangle_{8}\left\langle\left\langle\zeta_{\mathrm{s} . \mathrm{m} .}^{(\mathrm{PU})}\right\rangle_{8}\right.$ even for the heaviest NS, since $M_{c}^{\mathrm{DU}}$ is rather close to the maximum mass for our EoS. The MU and MMU contributions depend on the temperature as $\propto T^{6}$ and the PU and DU ones as $\propto T^{4}$. All contributions depend on the $r$-mode frequency as $\propto \omega^{-2}$. Pairing effects do not change the relative balance of partial contributions to $\zeta_{\text {s.m. }}$.

The energy can be dissipated not only via non-equilibrium soft-mode processes but also by the neutrino radiation. Hence, there is another source of the bulk viscosity, the radiative viscosity. The latter term was studied only recently in [28-30] for the MU and DU reactions only. We calculated contributions to the radiative bulk viscosity from MMU, PU and DU reactions. Smaller contributions come from the processes on weak neutral currents, such as nucleon bremsstrahlung reactions and nucleon pair breaking-formation processes in superfluid regions. We found that the radiative viscosity from MMU, PU and inmedium nucleon bremsstrahlung processes demonstrates a strong density dependence.
The shear viscosity contains several important contributions. These are the terms from the lepton $\eta_{l}$ and neutron-neutron scattering, $\eta_{n}$, and the neutrino contribution, $\eta_{\nu}$, existing for temperatures, when neutrinos are trapped in the star interior. The phonon contribution to the shear viscosity is found to be small for our choice of the pairing gaps.

The lepton shear viscosity term computed following ref. [8] proves to be by an order of magnitude smaller than the term, computed previously in $[9,31]$ and exploited in many papers studying $r$-modes. In spite of this suppression, the lepton contribution to the shear viscosity proves to be larger than the neutron one calculated with the free $N N$ cross-sections. In the regions with the proton pairing the lepton term is enhanced [8]. We incorporate the in-medium effects into $N N$ interaction amplitude and recalculate the nucleon shear viscosity $\eta_{n}$, which turns to be still smaller than the lepton one.

Neutrinos are trapped in the NS for temperatures above the opacity temperature $T_{\text {opac }}$ and, hence, can contribute to the shear viscosity. Such a contribution was not considered yet. Usually, $T_{\text {opac }}$ is defined as the temperature, above which the neutrino mean free path $\lambda_{\nu}(\bar{n}, T)$ at some averaged nucleon density $\bar{n}$ becomes less than the NS radius, $\lambda_{\nu}\left(\bar{n}, T_{\text {opac }}\right)=R$. Evaluation of the opacity temperature with the MU processes [22] yields $T_{\text {opac }} \simeq 22 \cdot 10^{9} \mathrm{~K}$ and this quantity weakly depends on the averaged density. The account for the MMU reactions leads to a decrease of $T_{\text {opac }}[11-14,10]$. The above definition of $T_{\text {opac }}$ does not take into account a strong density dependence of the MMU reactions, which makes possible that a dense interior is yet opaque and a broad outer part is already transparent for neutrinos. The radius of the region opaque for neutrinos is determined from the condition $\lambda_{\nu}\left(n\left(r_{\text {opac }}\right), T\right)=R-r_{\text {opac }}$. The opacity temperature can now be defined as the temperature, at which $r_{\text {opac }}=0$. The latter temperature proves to be significantly smaller than the opacity temperature introduced previously. We evaluate the neutrino shear viscosity term with account for the pionsoftening effects in the neutrino mean free path. Yet, for densities $n>n_{c}^{\pi}$ the pion condensation processes contribute to the neutrino shear viscosity and for the heaviest stars we add the contribution from the DU processes.

In fig. 2(b) we collect our results for shear and bulk viscosities averaged over the star density profiles, as they enter in times $\tau_{\eta}$ and $\tau_{\zeta}$. The viscosities are computed with account for the nucleon pairing and plotted as functions of the temperature for various star masses. The main contributions to the bulk viscosity come from the soft-mode and radiative terms. The main contribution to the shear viscosity is the lepton term for $T \lesssim 3 \cdot 10^{9} \mathrm{~K}$. It decreases with a temperature increase, and at $T \gtrsim 4 \cdot 10^{9} \mathrm{~K}$ the neutrino contribution starts dominating for middle-heavy and heavy neutron stars, whereas for the light neutron stars (with a mass $M \sim M_{\odot}$ ) it happens at $T \gtrsim 10^{10} \mathrm{~K}$. The bulk viscosity rises rapidly with growth of the temperature and the NS mass. At temperatures above $\sim 2 \cdot 10^{9}$ for all star masses the bulk viscosity exceeds the shear 

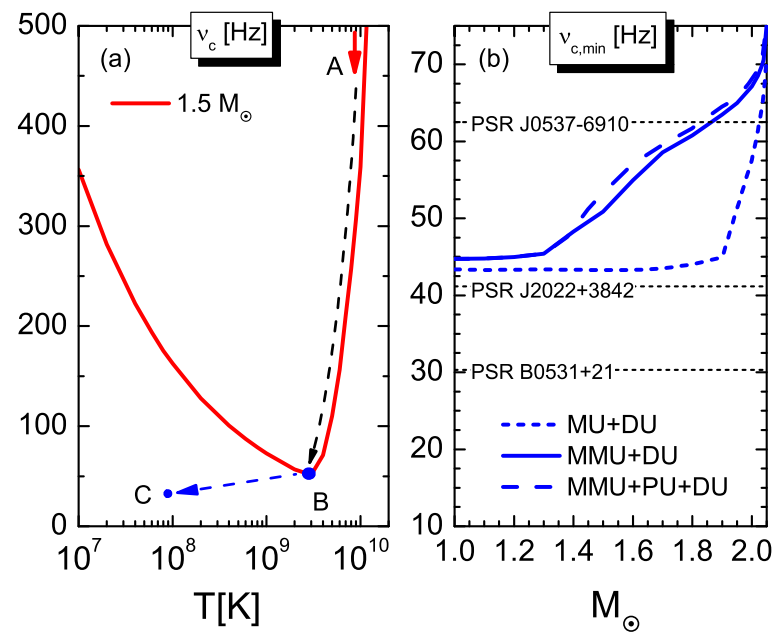

Fig. 3. Panel (a): Critical rotation frequency for the $r$-mode instability as a function of the star temperature for the star mass $1.5 M_{\odot}$. Panel (b): The minimal critical frequency of the star stable rotation (point B in panel (a)) as a function of the star mass, for different sets of reactions contributing to the bulk viscosity. Dotted lines show rotation frequencies of three most rapidly rotating pulsars.

viscosity. For the heaviest stars the averaged bulk viscosity is dominated by the $\mathrm{PU}$ and $\mathrm{DU}$ reactions, it exceeds the shear viscosity term already at $T>(2-5) \cdot 10^{8} \mathrm{~K}$.

The equation $\tau^{-1}\left(\nu_{\mathrm{c}}\right)=0$ determines the critical rotation frequency of a star. For frequencies larger than $\nu_{c}$ the $r$-modes amplitude, $a$, rapidly grows unless non-linear effects stabilize it at some maximal amplitude $a_{\max }$, for smaller frequencies the $r$-modes are not excited. The $r$ modes stability line on the temperature-frequency plane $\left(T, \nu_{\mathrm{c}}\right)$ with viscosities calculated within the nuclear medium cooling scenario is shown in fig. 3(a) for the star with the mass $1.5 M_{\odot}$. The newly born pulsar enters the plane in the upper-right corner (point A). The heat transport to the NS surface which includes the charged-lepton and nucleon thermal conductivity, neutrino opacity, reheating by the $r$-modes, etc., delays the cooling process. The star trajectory on the $\left(T, \nu_{c}\right)$ plane closely follows the critical line $\nu_{c}(T)$ between points A and B, provided the typical cooling time is larger than the spin-down time $\sim 100 \mathrm{~s} / a_{\max }^{2} \nu_{3}^{6}$, where $\nu_{3}=\nu / 10^{3} \mathrm{~Hz}$. For $a_{\max } \sim 1$ the point $\mathrm{B}$ with the frequency $\nu_{\mathrm{c}, \text { min }}$ corresponds to the minimum of $\nu_{\mathrm{c}}$ as a function of $T$. Cooling further down, the star enters into the $r$-mode stable region and its rotation frequency decreases at a much larger time scale determined by the magnetic dipole radiation (path from B to $\mathrm{C}$ in fig 3(a)). So, to explain the observed fast rotation frequency of the pulsar within this scenario the value of $\nu_{\mathrm{c} \text {,min }}$ must be larger than its current rotation frequency.

The quantity $\nu_{\mathrm{c}, \text { min }}$ is shown in fig. $3(\mathrm{~b})$ as a function of the pulsar mass for different sets of neutrino reactions contributing to the soft-mode bulk viscosity. Within the minimal cooling scenario when in-medium effects are not included (short dashed line in fig. $3(\mathrm{~b})), \nu_{\mathrm{c}, \text { min }}$ exceeds $\nu_{\max }^{\text {young }}$ only for the masses $M>2.03 M_{\odot}$ (when the DU reaction is already efficient), i.e. very close to the maxi- mum mass, $2.05 M_{\odot}$. However, if initially the star passes through the instability region, the developed $r$-modes blow off some part of the star matter. So, its final mass (in point B) can hardly be very close to the maximum mass. Alternatively, the experimental value of the frequency of the pulsar PSR J0537-6910 could be explained within the minimal cooling scenario, if one exploited EoS that allows for a lower DU threshold density. Reference [32] involving the data on $\dot{\nu}$ and $\nu$ presented arguments that within their analysis the curve AB in fig. 3(a) is shifted to the left for the relevant values of the amplitude $a_{\text {sat }}$, that could also allow to explain stability of PSR J0537-6910 within the minimal cooling paradigm. Within the nuclear medium cooling scenario, the DU processes are not needed to explain the stability of PSR J0537-6910. We explain it for $M>1.80 M_{\odot}$, if the MMU and PU processes are included or for $M>1.84 M_{\odot}$, if pion condensate is not formed.

Concluding, we recalculated contributions to the shear and bulk viscosities for all dissipation processes considered previously and we included new processes. We computed critical frequencies for the $r$-mode stability of rotating neutron stars. The in-medium polarization effects such as the Coulomb screening in the electron-muon plasma and the softening of pionic degrees of freedom with an increase of the nucleon density are incorporated. The latter effect is the same as used in the nuclear-medium cooling scenario applied successfully to the description of neutron star cooling $[7,15-17]$. The stability of the most rapidly rotating young pulsar PSR J0537-6910 is explained by the efficient MMU reactions provided its mass $M \geq 1.8 M_{\odot}$. Presence of the DU reactions is not required. Within the minimal cooling scenario the data on PSR J0537-6910 can be explained only, if EoS allows for the efficient DU processes. Finally, we should notice that there exist old pulsars in the LMXB sources, which have frequencies much higher than PSR J0537-6910. It is commonly believed that these pulsars are accelerated by accretion from the companion stars. They should be treated separately.

The work was supported by Grants VEGA 1/0457/12 and APVV-0050-11 and by "NewCompStar", COST Action MP1304. We thank Dr. Kai Schwenzer for discussions.

Open Access This is an open access article distributed under the terms of the Creative Commons Attribution License (http://creativecommons.org/licenses/by/4.0), which permits unrestricted use, distribution, and reproduction in any medium, provided the original work is properly cited.

\section{References}

1. P. Chatterjee, L. Hernquist, Astrophys. J. 543, 368 (2000).

2. F.E. Marshall et al., Astrophys. J. 603, 682 (2004).

3. N. Andersson, Astrophys. J. 502, 708 (1998).

4. J.L. Friedman, S.M. Morsink, Astrophys. J. 502, 714 (1998).

5. N. Andersson, K.D. Kokkotas, Int. J. Mod. Phys. D 10, 381 (2001).

6. L. Lindblom et al., Phys. Rev. Lett. 80, 4843 (1998). 
7. D. Blaschke et al., Phys. Rev. C 88, 065805 (2013).

8. P.S. Shternin, D.G. Yakovlev, Phys. Rev. D 78, 063006 (2008).

9. E. Flowers, N. Itoh, Astrophys. J. 230, 847 (1979).

10. A.B. Migdal et al., Phys. Rep. 192, 179 (1990).

11. D.N. Voskresensky, A.V. Senatorov, Sov. Phys. JETP 63, $885(1986)$

12. A.V. Senatorov, D.N. Voskresensky, Phys. Lett. B $\mathbf{1 8 4}$ 119 (1987).

13. C. Schaab et al., Astron. Astrophys. 321, 591 (1997).

14. D.N. Voskresensky, Lect. Notes Phys. 578, 467 (2001).

15. D. Blaschke et al., Astron. Astrophys. 424, 979 (2004).

16. H. Grigorian, D.N. Voskresensky, Astron. Astrophys. 444, 913 (2005).

17. D. Blaschke et al., Phys. Rev. C 85, 022802 (2012).

18. A. Akmal et al., Phys. Rev. C 58, 1804 (1998).

19. P. Demorest et al., Nature 467, 1081 (2010).
20. J. Antoniadis et al., Science 340, 6131 (2013).

21. P. Haensel, A.Y. Potekhin, Astron. Astrophys. 428, 191 (2004).

22. B.L. Friman, O.V. Maxwell, Astrophys. J. 232, 541 (1979)

23. A.B. Migdal, Theory of Finite Fermi Systems and Properties of Atomic Nuclei (Wiley and Sons, New York, 1967).

24. A. Schwenk, B. Friman, Phys. Rev. Lett. 92, 082501 (2004).

25. D. Page et al., Nucl. Phys. A 777, 497 (2006).

26. R.F. Sawyer, Phys. Rev. D 39, 3804 (1989).

27. P. Haensel et al., Astron. Astrophys. 372, 130 (2001).

28. B.A. Sa'd, J. Schaffner-Bielich, arXiv:0908.4190.

29. S.-H. Yang et al., Phys. Lett. B 683, 255 (2010).

30. S.-H. Yang et al., Mon. Not. R. Astron. Soc. 403, 2007 (2010).

31. C. Cutler, L. Lindblom, Astrophys. J. 314, 234 (1987).

32. M.G. Alford, K. Schwenzer, Astrophys. J. 781, 26 (2014). 